\title{
Molecular order and disorder of surfactants in clay nanocomposites $\dagger \neq$
}

\author{
Tim J. Tambach, ${ }^{* a}$ Edo S. Boek ${ }^{b}$ and Berend Smit ${ }^{a c}$ \\ Received 27th January 2006, Accepted 21st April 2006 \\ First published as an Advance Article on the web 8th May 2006 \\ DOI: $10.1039 / b 601373 b$
}

\section{Disorder of intercalated surfactant molecules in clay minerals causes gradual swelling, rather than commonly assumed swelling in discrete steps.}

Nanocomposites of clay minerals and surfactants are of practical importance in many fields. ${ }^{1}$ Beside the use of these systems for pollution removal from waste water, ${ }^{2}$ they can also be used as catalysts, ${ }^{3}$ to stabilize clay formations while drilling for hydrocarbons, ${ }^{4}$ or for improving the strength of polymers. ${ }^{5,6}$ The nanocomposites are obtained through exchange of natural counter ions by ionic surfactants, such as alkylammonium. ${ }^{7}$ Despite the importance of such nanocomposites, their behaviour is not understood. It is commonly assumed that the distance between the clay platelets (basal spacing) increases stepwise or linearly with increasing alkylammonium chain length. ${ }^{8}$ The traditional explanation for this observation is that the surfactants form all-trans conformations between the clay platelets, ${ }^{8}$ either in layers or tilted in a liquid crystalline ordered phase. However, this explanation has recently been debated. ${ }^{9}$ X-Ray diffraction measurements show a complex intercalation behaviour. ${ }^{7}$ For example, when basal spacings are measured, corresponding to values in-between monolayer and bilayer structures, then these values are usually attributed to layer charge heterogeneity. ${ }^{10}$ In this case, the explanation is that different layer charges within a clay sample cause a varying surfactant loading in the interlayers between two clay platelets and therefore an ensemble of monoand bilayer structures, but this has never been directly confirmed experimentally. As X-ray diffraction peaks for such states overlap, the resulting peak is broad and difficult to interpret.

Molecular simulations are ideally suited for obtaining more insight in the intercalation behaviour of surfactants in clay minerals. Several simulation studies have been carried out to compute the basal spacing for some clay minerals and some alkylammonium surfactants. ${ }^{6,11-13}$ These simulation studies show increasing disorder (phase transitions) of the surfactants in mica clay minerals with increasing temperature. ${ }^{6,12}$ Our simulations are complementary to the previous studies, in the sense that they cover an extensive range of surfactant chain

\footnotetext{
${ }^{a}$ Van't Hoff Institute for Molecular Sciences, University of Amsterdam, Nieuwe Achtergracht 166, 1018 WV Amsterdam, The Netherlands.E-mail: tim.tambach@tno.nl

${ }^{b}$ Schlumberger Cambridge Research, High Cross, Madingley Road, Cambridge, UK CB3 OEL. E-mail: boek@cambridge.oilfield.slb.com ${ }^{c}$ CECAM (Centre Européen de Calcul Atomique Moléculaire, Ecole Normale Supérieure), 46 Allée d'Italie, 69364 Lyon Cedex 7. France.E-mail:bsmit@cecam.fr

$\dagger$ The HTML version of this article has been enhanced with additional colour images.

$\ddagger$ Electronic supplementary information (ESI) available: Details of our clay models, force field and molecular simulation methods. See DOI: $10.1039 / \mathrm{b} 601373 \mathrm{~b}$
}

lengths. Also, in contrast with mica, we consider clay minerals that are able of swelling. We find, for the first time, full quantitative agreement between the experimental and simulated swelling behaviour of alkylammonium in montmorillonite and vermiculite clay minerals. Based on our simulations, we give a new explanation for the gradual increase of the clay platelets distance. We show evidence that this is a true physical phenomenon, caused by disorder of the surfactant molecules.

Out of several available molecular clay models, ${ }^{6,14,15}$ we choose a clay model ${ }^{15}$ that has proven to be successful in predicting the basal spacing of natural clay minerals with water, ${ }^{15,16}$ the swelling capabilities of clay minerals, ${ }^{17,18}$ and hysteresis in clay swelling. ${ }^{17,19}$ In our simulations, we use molecular dynamics to move the flexible alkylammonium molecules with a time step $t$ of $1.0 \mathrm{fs}$ at a constant temperature $T$ of $298 \mathrm{~K}$ using the Nosé-Hoover thermostat. The SHAKE algorithm is included to constrain all bond lengths ${ }^{20}$ of the alkylammonium molecules. We describe the interactions between alkylammonium molecules and the clay mineral using the OPLS forcefield. ${ }^{21}$ For a correct sampling of the phase space of the long-chain alkylammonium molecules, we found it essential to use the configurational bias Monte Carlo scheme. ${ }^{22}$ Our simulations are performed in the isobaric-isothermal $\left(\mathrm{NP}_{z} \mathrm{~T}\right)$ ensemble, with $P_{z}=1.0 \mathrm{~atm}$. The basal spacing changes are carried out with a Monte Carlo algorithm, ${ }^{22}$

$$
\begin{aligned}
\operatorname{acc}(\mathrm{o} & \rightarrow \mathrm{n}) \\
& =\min \left(1, \exp \left[\frac{-\left(\Delta U+P_{z} \Delta V\right)}{k T}+N_{a a} \ln \left(\frac{L_{z}(\mathrm{n})}{L_{z}(\mathrm{o})}\right)\right]\right)
\end{aligned}
$$

where acc $(\mathrm{o} \rightarrow \mathrm{n})$ denotes the acceptance probability of going from an old (o) to a new (n) configuration. $\Delta U$ and $\Delta V$ are the difference in potential energy and volume, respectively, between the new and the old configuration. $k$ represents the Boltzmann constant. The number of alkylammonium molecules is given by $N_{a a}$ and the $z$-dimensions of the new and old configurations are defined as $L_{z}(\mathrm{n})$ and $L_{z}(\mathrm{o})$, respectively. We investigate three types of clay minerals: Wyoming montmorillonite, Cheto montmorillonite, and Vermiculite. In this order, these clays have a systematically increasing negative layer charge ${ }^{7}$ of $0.625 \mathrm{e}, 0.875 \mathrm{e}$, and 1.3125 e per unit-cell, respectively. In our simulations, one clay platelet consists of 32 unit-cells with $L_{x}=42.24 \AA$ and $L_{y}=36.56 \AA$, corresponding to the dimensions in the $x$ and $y$ directions, respectively. The 32 unit-cells of a clay layer imply a charge of 20, 28, and 42 e for Wyoming montmorillonite, Cheto montmorillonite, and Vermiculite, respectively, which is compensated by an equivalent amount of alkylammonium molecules in each interlayer. More details on our models are 


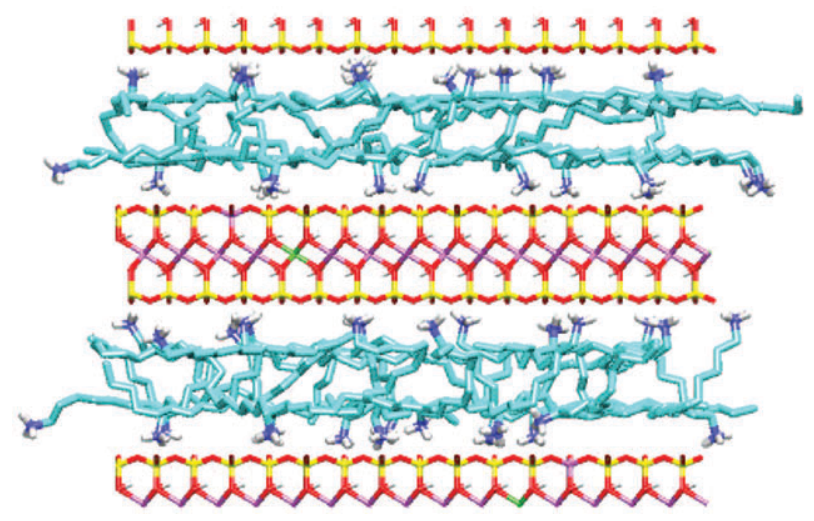

Fig. 1 Snapshot of intercalated surfactants (butadecylammonium; $\mathrm{H}_{3} \mathrm{~N}^{+} \mathrm{C}_{14} \mathrm{H}_{29}$ ) in the interlayer space of Cheto montmorillonite. The snapshot shows a bilayer structure with a basal spacing of $17.6 \AA$. In the clay crystal, $\mathrm{O}=$ red, $\mathrm{H}=$ silver, $\mathrm{Si}=$ yellow, $\mathrm{Al}=$ purple, $\mathrm{Mg}=$ green. For the surfactants, $\mathrm{N}=$ blue, $\mathrm{H}=$ silver, and $\mathrm{C}=$ cyan.

given in the ESI† and in ref. 23. A typical snapshot of the system is shown in Fig. 1.

Our simulations in the isobaric-isothermal allow us to measure the basal spacing of the intercalated clay systems from ensemble averages, as a function of increasing chain length of the alkylammonium ions, for the three different clays detailed above. The results are shown in Fig. 2. We find that the simulated basal spacings, as a function of the carbon chain length, are in excellent agreement with experimental data. ${ }^{7} \mathrm{We}$ observe that the swelling behaviour strongly depends on the composition of the clay mineral. In the following, we will show that the differences in swelling behaviour can be explained in terms of conformational differences of the surfactant molecules.

For Wyoming montmorillonite, with a low coverage of alkylammonium ions, we observe that monolayers are stable

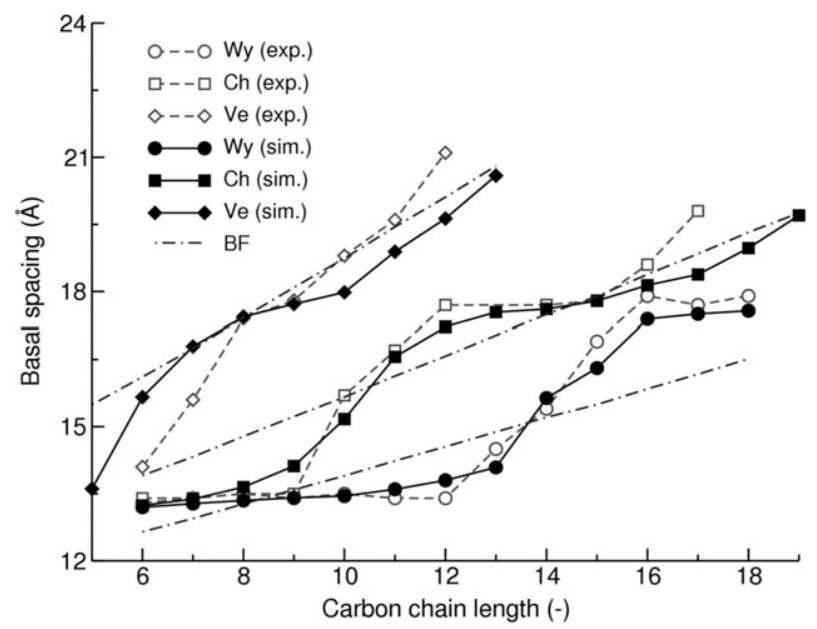

Fig. 2 Basal spacing as a function of the alkylammonium carbon chain length for Wyoming montmorillonite (Wy), Cheto montmorillonite (Ch), and Vermiculite (Ve). The closed symbols represent our simulation results and the open symbols are experimental data. ${ }^{7}$ We computed the bulk fluid densities of normal alkanes to estimate the basal spacing of a clay system where the alkylammonium molecules would behave as a bulk fluid (BF). The error bars of our simulation results are smaller than the symbol size and the lines between the data points are to guide the eye. up to a carbon chain length of 13 . The corresponding basal spacing is approximately $13.6 \AA$. Chains with 16 to 18 carbon atoms form bilayers with a basal spacing of $17.6 \AA$. In-between the mono- and bilayer domains, we find intermediate basal spacings. Cheto montmorillonite, with a higher alkylammonium coverage, shows a more gradual mono- to bilayer transition, which starts at a shorter chain length in comparison with Wyoming montmorillonite. Stable mono- and bilayers almost disappear in the case of Vermiculite with the highest layer charge and alkylammonium loading. This results in an almost continuous increase of the basal spacing as a function of the carbon chain length. Our simulation results confirm the experimental observations for both low and high clay layer charges. We recall that the common belief is that clay minerals with a relatively low loading and layer charge show a stepwise behaviour from mono- to bilayers. ${ }^{7,8}$ On the other hand, a linear increase of the basal spacing with increasing carbon chain length is assumed for clay minerals with a high layer charge and loading. Recent experimental results support our simulated smooth curves. ${ }^{24}$ As a reference, Fig. 2 also shows estimates of the basal spacing for the hypothetical case that alkylammonium would behave as a bulk fluid between the clay platelets, using computed bulk fluid densities of alkanes. Interestingly, this model nicely reproduces the experimental and simulated curves for Vermiculite with the highest loading, but deviates significantly for the other two clay minerals with lower layer charge.

We can explain the difference in the behaviour of the three clay minerals, using the average percentage of gauche orientations in the alkylammonium chains (Fig. 3). In the bulk fluid reference, approximately $25 \%$ of the chains have gauche orientations, close to the value that is normally observed in liquid alkanes. ${ }^{9}$ We show that oscillations in the distribution of gauche conformations occur for surfactants in clay nanocomposites. This indicates a fluctuating order and disorder of the alkylammonium molecules. We recall that, up till now, the surfactants are usually considered to form all-trans

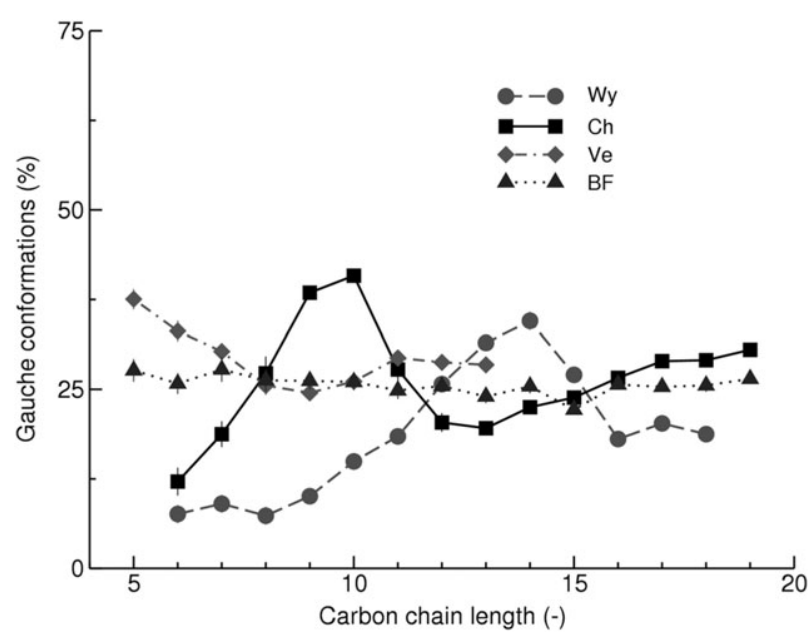

Fig. 3 Average percentage of gauche conformations in the alkylammonium molecules as a function of the carbon chain length for Wyoming montmorillonite (Wy), Cheto montmorillonite (Ch), and Vermiculite (Ve). As a reference we included the percentage of gauche conformations in the bulk fluid (BF). Most error bars are smaller than the symbol size and the lines between the data points are to guide the eye. 


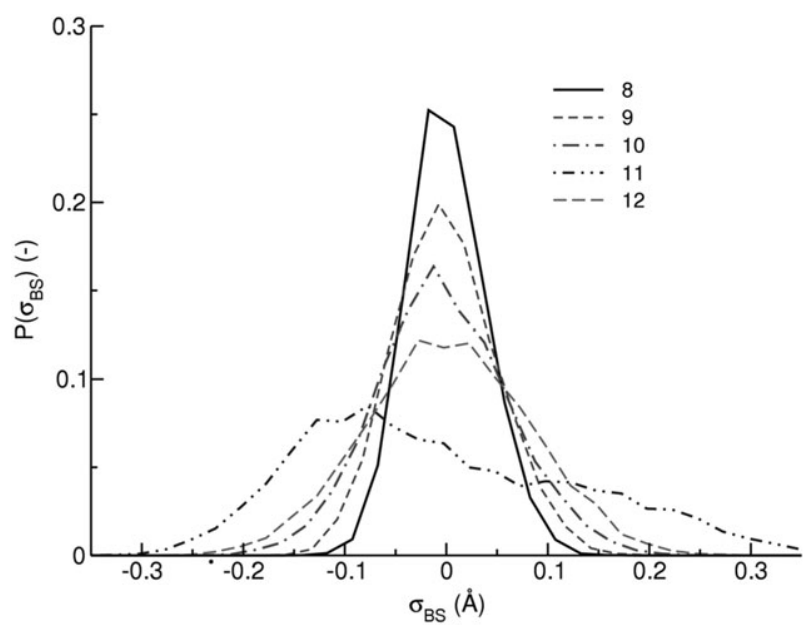

Fig. 4 Distribution of the basal spacing fluctuations $\left(\mathrm{P}\left(\sigma_{\mathrm{BS}}\right)\right)$ for Cheto montmorillonite and alkylammonium carbon chain lengths of 8 to 12 .

conformations between the clay platelets. ${ }^{8}$ Recent molecular simulation work, on the other hand, shows that the surfactant molecules display a distribution of gauche and trans conformations. ${ }^{6,13,25}$ Here, we observe that the occurrence of gauche conformations in the system is related to the basal spacing (Fig. 2). Mono- and bilayer configurations in the clay systems have a smaller percentage of gauche conformations than the bulk reference. This implies more ordering due to confinement. On the other hand, the transition domains from mono- to bilayers have a relatively high percentage of gauche conformations. Consequently, these structures are more disordered and have more fluid-like properties. To investigate this point in more detail, we carried out additional simulations where the molecules are artificially constrained in all-trans configurations (not shown). Interestingly, we do observe a stepwise increase of the spacing in the artificially constrained case. This confirms our explanation suggested above. The observation of more fluidlike properties in the transition domain is supported by the sampling of the basal spacing fluctuations. Fig. 4 shows these fluctuations for Cheto montmorillonite. We find that the distribution of basal spacings is broader in the transition domain. This indicates that fluctuations occur over a larger domain of the basal spacing. This, in a qualitative sense, also implies a higher compressibility. Stronger fluctuations become especially apparent in the case of a carbon chain length of 11 . We suggest that the increased fluidity in the transition domain may be considered as an alternative explanation for the broadening of X-ray diffraction peaks, commonly observed experimentally. ${ }^{10,23}$

From the results described above, we conclude that at small clay layer spacings, the confinement induces a (limited) molecular ordering of the alkylammonium ions. At larger clay layer spacings, on the other hand, the density and molecular configurations of the systems resemble those of ordinary bulk fluids. Our simulations confirm the experimental X-ray measurements of the clay basal spacing as a function of increasing alkylammonium chain length, for three different clays with increasing layer charge. The results can be explained in terms of different distributions of gauche-trans conformations of the surfactants. We find that a gradual increase of the basal spacing is physically stable. This can be explained by a complex disordering of the alkylammonium molecules, resulting in more fluidlike behaviour.

\section{Acknowledgements}

This work is supported by the Netherlands Research Council for Chemical Sciences (NWO-CW) through PIONIER. We thank Dr M. Schenk, Dr P. G. Bolhuis, and Dr D. A. Laird for helpful discussions.

\section{References}

1 (a) A. Weiss, Angew. Chem., Int. Ed., 1963, 2, 134-144; (b) B. K. G. Theng, The Chemistry of Clay-Organic Reactions, Adam Hilger, London, 1974.

2 (a) S. A. Boyd and W. F. Jaynes, in CMS Workshop Lectures. Layer Charge Characteristics of 2:1 Silicalite Minerals, ed. A. R. Mermut, The Clay Minerals Society, Boulder, CO, Editon edn., 1993, vol. 6, pp. 48-77; (b) M. Ogawa, T. Aono, K. Kuroda and C. Kato, Langmuir, 1993, 9, 1529-1533; (c) M. J. Carrizosa, P. J. Rice, W. C. Koskinen, I. Carrizosa and M. C. Hermosin, Clays Clay Miner., 2004, 52, 341-349.

3 T. J. Pinnavaia, Science, 1983, 220, 365-371.

4 E. S. Boek, P. V. Coveney, B. Craster and P. Reid, in Chemicals in the oil industry, Proc. of the 6th Int. Symp. on Chemistry in the Oil Industry, Royal Society of Chemistry, 1998, pp. 58-70.

5 (a) C. Calberg, R. Jérome and J. Grandjean, Langmuir, 2004, 20 , 2039-2041; (b) T. D. Fornes, D. L. Hunter and D. R. Paul, Macromolecules, 2004, 37, 1793-1798.

6 H. Heinz, H. J. Castelijns and U. W. Suter, J. Am. Chem. Soc., 2003, 125, 9500-9510.

7 D. A. Laird, A. D. Scott and T. E. Fenton, Clays Clay Miner., 1989, 37, 41-46.

8 G. Lagaly and A. Weiss, Determination of the layer charge in mica-type layer silicates, , in Proc. of the Int. Clay Conf., held in Tokyo, ed. L. Heller, Israel University Press, Jerusalem, 1969, vol. 1, pp. 61-80.

9 R. A. Vaia, R. K. Teukolsky and E. P. Giannellis, Chem. Mater., 1994, 6, 1017-1022.

10 D. A. Laird, in CMS Workshop Lectures. Layer Charge Characteristics of 2:1 Silicalite Minerals, ed. A. R. Mermut, The Clay Minerals Society, Boulder, CO, 1993, vol. 6, pp. 80-103.

11 Q. H. Zeng, A. B. Yu, G. Q. Lu and R. K. Standish, J. Phys. Chem. $B, 2003,108,10025-10033$.

12 H. Heinz and U. W. Suter, Angew. Chem., Int. Ed., 2004, 43, 2239-2243.

13 E. Hackett, E. Manias and E. P. Giannelis, J. Chem. Phys., 1998, 108, 7410-7415.

14 R. T. Cygan, J.-J. Liang and A. G. Kalinichev, J. Phys. Chem. B, 2004, 108, 1255-1266.

15 N. T. Skipper, F.-R. C. Chang and G. Sposito, Clays Clay Miner., 1995, 43, 285-293.

16 M. Chávez-Páez, K. Van Workum, L. dePablo and J. J. dePablo, J. Chem. Phys., 2001, 114, 1405-1413.

17 E. J. M. Hensen and B. Smit, J. Phys. Chem. B, 2002, 106, 12664-12667.

18 (a) E. S. Boek, P. V. Coveney and N. T. Skipper, Langmuir, 1995, 11, 4629-4631; (b) E. S. Boek, P. V. Coveney and N. T. Skipper, J. Am. Chem. Soc., 1995, 117, 12608-12617.

19 T. J. Tambach, P. G. Bolhuis and B. Smit, Angew. Chem., Int. Ed., 2004, 43, 2650-2652.

20 M. P. Allen and D. J. Tildesley, Computer simulation of liquids, Clarendon Press, Oxford, 1987.

21 W. L. Jorgensen, J. D. Madura and C. J. Swenson, J. Am. Chem. Soc., 1984, 106, 6638-6646.

22 D. Frenkel and B. Smit, Understanding Molecular Simulation: from Algorithms to Applications, Academic Press, San Diego, 2nd edn, 1996.

23 T. J. Tambach, PhD thesis, Universiteit van Amsterdam, 2005.

24 M. Janek and L. Smrcok, Clays Clay Miner., 1999, 47, 113-118.

25 Q. H. Zeng, A. B. Yu, G. Q. Lu and R. K. Standish, J. Phys. Chem. B, 2004, 108, 10025-10033. 